\title{
evaluación de la resistencia de pilares de hormigón por medio de testigos cortados: nuevas aportaciones
}

\author{
J. L. RAMIREZ ORTIZ, Prof. Dr. ingeniero industrial
}

J. M. BARCENA DIAZ, ingeniero industrial

\begin{abstract}
sinopsis El presente trabajo es continuación del aparecido en esta Revista, número 255, noviembre de 1973, sobre el tema del control de hormigón endurecido por medio de probetas testigo cortadas con trépano.

Se expone el total de los resultados obtenidos en los diferentes ensayos en forma de cuadros y gráficos, habiéndose utilizado para las pruebas pilares de tamaño estructural.

Los temas estudiados son, en primer lugar, el de la representatividad de la resistencia de los testigos cortados respecto a la del pilar de que se toman y la variación de dicha resistencia con la humedad y la altura de extracción sobre la base del pilar.

Como segundo tema se analiza la pérdida de resistencia de pilares a los que después de la perforación de un testigo de $10 \mathrm{~cm}$ de diámetro, se les rellena por diferentes métodos el agujero causado por dichá operación.

La investigación ha sido realizada en los laboratorios de la Cátedra de Estructuras de la Escuela Superiór

\section{INTRODUCCION}

Cuando se realiza inspección de obras de hormigón endurecido $y$, sobre todo, cuando se descubren elementos estructurales de baja calidad, surgen algunos interrogantes en cuanto a la validez de los ensayos efectuados y a la interpretación de los resultados obtenidos.

En un trabajo anterior (INFORMES DE LA CONSTRUCCION núm. 255, noviembre 1973) exponíamos una serie de resultados obtenidos en torno a dos problemas. El primero era el del deterioro físico que experimenta la probeta extraída de hormigón endurecido por efecto del corte de la corona empleada. El segundo era el de la valoración de la eficacia resistente de diversos métodos de relleno de los agujeros cilíndricos hechos en pilares por la extracción de testigos para control de resistencia.

Como continuación de estos trabajos exponemos a continuación los resultados y conclusiones obtenidos a partir de la nueva experimentación desarrollada.

Una de las líneas de trabajo ha sido continuación del estudio del segundo de los problemas, anteriormente citados, conducente a comprobar cómo queda desde el punto de vista resistente un pilar de tamaño normal, al que después de extraerle una probeta con trépano a mitad de su altura, se rellena el hueco con hormigón de calidad con un aditivo expansivo. En la presente ocasión el pilar ha sido de $2 \mathrm{~m}$ de altura y el relleno, de acuerdo con la anterior investigación, se ha realizado con hormigón normal de buena resistencia con expansivo, que es lo que dio mejores resultados en el trabajo antes citado.

La segunda línea de trabajo ha consistido en ponderar el valor de los testigos extraídos por corte en función de su altura en el pilar y su comparación con la resistencia del pilar mismo y con la de probetas moldeadas.

\section{PLANTEAMIENTO Y DESARROLLO DE LA INVESTIGACION}

\subsection{Propósito y plan de trabajo}

Los diferentes aspectos que se han querido tocar en la presente ocasión han sido los siguientes:

a) Relación entre resistencias de probetas cilíndricas moldeadas, testigos extraídos por corte y pilares de tamaño natural, todo procedente del mismo hormigón. 
b) Influencia en la resistencia de los testigos extraídos por corte de la altura del pilar a que se han tomado.

c) Influencia en la resistencia de los testigos de la saturación de agua previa a la rotura.

d) Eficacia resistente de pilares a los que se ha practicado un agujero para la extracción del testigo que posteriormente se rellena.

e) Influencia en los apartados anteriores del nivel resistente del hormigón de que se trate.

Para poder obtener conclusiones relativas a los puntos anteriores la preparación de probetas y piezas de ensayo se ha desarrollado como sigue:

- Se ha trabajado con dos niveles ceóricos de resistencia: 90 y $130 \mathrm{kp} / \mathrm{cm}^{2}$. Son valores muy bajos y el primero fuera de Normas, pero estimamos interesante conocer lo que pasa con los malos hormigones que es en los que se presenta como más importante el determinar con mayor exactitud la validez y representatividad de los ensayos, debido al menor margen de seguridad y mayor dispersión que ofrecen.

- Por cada nivel de resistencia se han realizado seis masas, con cada una de las cuales se han moldeado 12 probetas cilíndricas de $15 \times 30 \mathrm{~cm}$ y tres pilares de sección transversal de $25 \times 25 \mathrm{~cm}$ y $2 \mathrm{~m}$ de altura, con una armadura mínima sólo para evitar su rotura durante el manejo de los mismos.

- De cada grupo de tres pilares uno se perforaba a mitad de su altura, a los 60 días, rellenándose a continuación el agujero practicado. De otro se extraían a diferentes alturas 20 testigos cilíndricos de $10 \mathrm{~cm}$ de diámetro. El tercero quedaba tal como se moldeó. En la figura 1 están representadas todas las probetas correspondientes a una masa.

- Por ello, después de rotas todas las piezas y probetas hemos dispuesto de 36 resultados correspondientes a pilares; 144 , a probetas cilíndricas moldeadas de $15 \times 30$ centímetros, y 240 , a testigos cilíndricos de $10 \times 20 \mathrm{~cm}$, cortados con trépano, a partir de los cuales se han desarrollado las conclusiones.
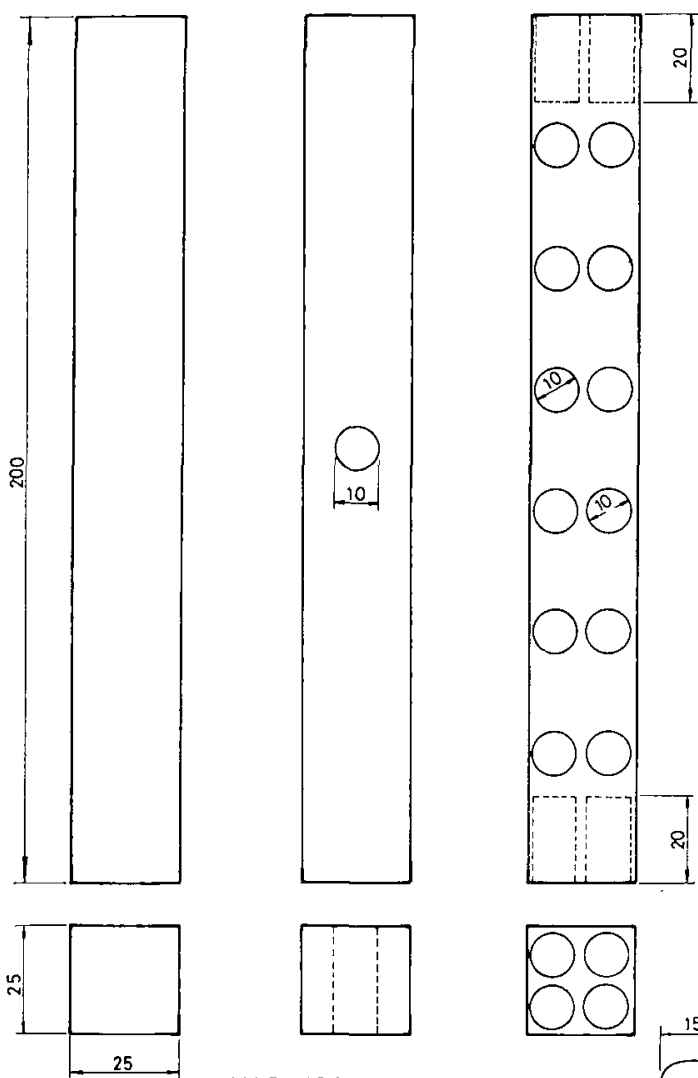

HORMIGONADO VERTICAL

+12 PROBETAS CILINDRICAS

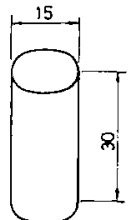

Fig. 1. Piezas y probetas procedentes de una masa.

\subsection{Datos técnicos correspondientes a los ensayos}

\subsubsection{Aridos}

Se han utilizado los áridos de machaqueo calizos habituales de nuestra región. El tamaño máximo ha sido de $30 \mathrm{~mm}$, habiéndose empleado tres fracciones: guijo, guijillo y arena.

El contenido en finos (UNE 7 135) de la arena ha sido del $12 \%$, valor muy superior al $5 \%$ que tolera la Instrucción Española de Hormigón, pero muy frecuente en gran parte de las canteras de caliza de la zona.

La mezcla óptima se ha realizado con el criterio de ajustarla lo más posible a la fórmula de Fuller $y$ ha resultado la siguiente, expresada en peso:

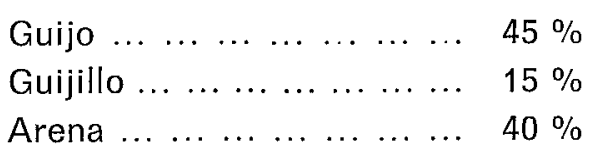




\begin{tabular}{|c|c|c|}
\hline CARACTERISTICAS & Masa de $90 \mathrm{kp} / \mathrm{cm}^{2}$ & Masa de $130 \mathrm{kp} / \mathrm{cm}^{2}$ \\
\hline 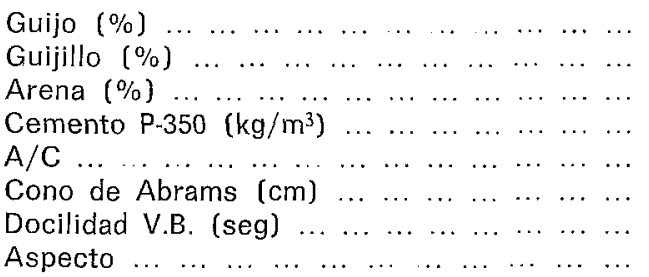 & $\begin{array}{l}45 \\
15 \\
40 \\
200 \\
1,30 \\
17 \\
\text { Muy fluida }\end{array}$ & $\begin{array}{l}45 \\
15 \\
40 \\
200 \\
1,08 \\
5 \\
3 \\
\text { Plástica }\end{array}$ \\
\hline
\end{tabular}

\subsubsection{Confección y conservación de probetas y testigos}

El hormigón de la masa de $90 \mathrm{~kg} / \mathrm{cm}^{2}$ ha sido asentado a barra. El de la de $130 \mathrm{~kg} / \mathrm{cm}^{2}$ ha sido vibrado.

De las 12 probetas cilíndricas moldeadas en el curso del hormigonado de los pilares correspondientes a una masa, 6 se han conservado en agua a $20 \pm 1^{\circ} \mathrm{C}$ de temperatura y otras 6 al aire ambiente. Los 3 pilares de $25 \times 25 \times 200 \mathrm{~cm}$ preparados con cada masa se conservaron al aire.

Uno de ellos se reservaba sin ninguna modificación hasta la rotura. Al segundo se le extraía con trépano a mitad de su altura, a la edad de 60 días, un testigo de $10 \mathrm{~cm}$ de diámetro que le atravesaba de parte a parte. A continuación se rellenaba con una masa de hormigón, con un aditivo expansivo, de las siguientes características:

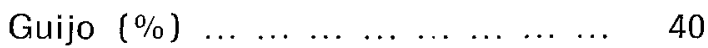

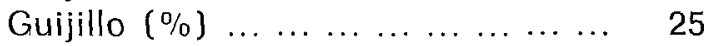

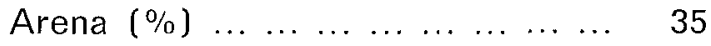

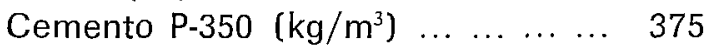

Aditivo (\%o respecto al cemento) ... 9

$\begin{array}{lllllllllll}A / C & \ldots & \ldots & \ldots & \ldots & \ldots & \ldots & \ldots & \ldots & \ldots & \ldots\end{array}$

Consistencia $\ldots \ldots \ldots \ldots \ldots$ Plástica blanda

La resistencia media de probetas hechas con este material de relleno fue a 90 días de $250 \mathrm{kp} / \mathrm{cm}^{2}$.

Del tercer pilar se extrajeron por corte, a la edad de 145 días, 20 probetas de $10 \mathrm{~cm}$ de diámetro en las posiciones indicadas en la figura 1. De las extraídas perpendicularmente a la directriz del pilar, 6 se conservaban al aire hasta la rotura y otras 6 se sumergían en agua 48 horas antes para romperlas saturadas de agua. Las 8 extraídas paralelamente a la dirección del pilar en sus extremos superior e inferior se mantuvieron igualmente al aire hasta el momento de rotura.

El aire ambiente de conservación de probetas y pilares, exceptuando las probetas moldea- das conservadas en agua, fue de una temperatura de $20 \pm 1^{\circ} \mathrm{C}$ y una humedad superior a $80 \%$. Todas las probetas y pilares procedentes de la misma masa se conservaron en total proximidad.

La extracción de probetas por corte se ha realizado con un aparato rotativo que gira en carga a 730 r.p.m., siendo las coronas cortantes del tipo de polvo de diamante.

Los ensayos de rotura a compresión se hicieron a la edad de 150 días para todas las piezas y probetas. (El hormigón de relleno del agujero del segundo pilar tenía en ese momento una edad de 90 días) .

Los ensayos de probetas moldeadas y testigos cortados se han efectuado en dos prensas Amsler, una de $500 \mathrm{Mp}$ y otra de $50 \mathrm{Mp}$. Los pilares se han ensayado en una prensa de $250 \mathrm{Mp}$ que permite romper piezas hasta de $2 \mathrm{~m}$ de altura. Por medio de un cadenciómetro se ha regulado la velocidad de aplicación de la carga a $5 \mathrm{kp} / \mathrm{cm}^{2} \cdot \mathrm{s}$.

El conjunto de resultados que se han obtenido de los diferentes ensayos para cada calidad de hormigón, comprende:

- Resistencias de pilares de $25 \times 25 \times 200$ centímetros sin perforar.

- Resistencias de pilares de $25 \times 25 \times 200$ centímetros con perforaciones de $10 \mathrm{~cm}$ de diámetro rellenas con hormigón expansivo.

- Resistencias de probetas cilíndricas normales moldeadas conservadas en agua 0 al aire.

- Resistencias de testigos cilíndricos de $10 \times 20 \mathrm{~cm}$ extraídos de un pilar a diferentes alturas y rotos en seco y saturados de agua.

\subsubsection{Resultados de los ensayos de compresión}

Aparecen recogidos en los cuadros 1,2, 3 y 4 , de los que se han sacado las siguientes conclusiones: 
CUADRO 1

MASA DE $90 \mathrm{kp} / \mathrm{cm}^{2}$

[Resistencia a la compresión en $\mathrm{kp} / \mathrm{cm}^{2}$ ]

\begin{tabular}{|c|c|c|c|c|}
\hline \multirow[t]{2}{*}{ Masa } & \multicolumn{2}{|c|}{$\begin{array}{c}\text { Pilares moldeados } \\
25 \times 25 \mathrm{~cm} \\
\mathrm{~h}=2 \mathrm{~m}\end{array}$} & \multicolumn{2}{|c|}{$\begin{array}{l}\text { Probetas cilíndricas moldeadas } \\
\qquad \begin{array}{c}\phi=15 \mathrm{~cm} \\
h=30 \mathrm{~cm}\end{array}\end{array}$} \\
\hline & $\begin{array}{l}\text { Sin } \\
\text { perforar }\end{array}$ & $\begin{array}{c}\text { Perforado y } \\
\text { relleno }\end{array}$ & $\begin{array}{l}\text { Conservadas al aire } \\
20 \pm 1^{\circ} \mathrm{C}>80 \% \mathrm{HR}\end{array}$ & $\begin{array}{c}\text { Conservadas en agua } \\
20 \pm 1^{\circ} \mathrm{C}\end{array}$ \\
\hline 1 & 87,0 & 90,5 & $\begin{array}{r}102,0-65,5-77,5 \\
77,0-69,5-76,5\end{array}$ & $\begin{array}{l}98,5-78,0-69,0 \\
69,0-72,5-83,5\end{array}$ \\
\hline 2 & 72,5 & 66,5 & $\begin{array}{l}61,0-63,5-72,0 \\
94,5-66,5-81,0\end{array}$ & $\begin{array}{l}73,0-69,5-70,5 \\
85,0-80,0-83,0\end{array}$ \\
\hline 3 & 90,0 & 80,5 & $\begin{array}{l}75,5-83,0-90,5 \\
77,5-74,5-86,5\end{array}$ & $\begin{array}{l}79,0-86,0-81,5 \\
81,0-79,5-97,0\end{array}$ \\
\hline 4 & 84,0 & 58,0 & $\begin{array}{l}70,0-77,5-63,5 \\
72,0-82,5-77,5\end{array}$ & $\begin{array}{l}68,0-68,0-70,0 \\
88,5-84,5-82,5\end{array}$ \\
\hline 5 & 76,8 & 75,2 & $\begin{array}{l}98,5-64,0-75,5 \\
69,7-66,2-81,0\end{array}$ & $\begin{array}{l}91,2-69,0-74,2 \\
66,8-66,8-81,0\end{array}$ \\
\hline 6 & 84,0 & 73,5 & $\begin{array}{r}112,0-61,0-75,0 \\
80,5-72,5-81,0\end{array}$ & $\begin{array}{r}111,0-66,5-83,0 \\
88,5-78,0-77,5\end{array}$ \\
\hline $\begin{array}{l}\text { Valor } \\
\text { medio }\end{array}$ & 82,4 & 74,0 & 77,0 & 79,2 \\
\hline $\begin{array}{c}\text { Coef. var. } \\
{[\%]}\end{array}$ & 7,9 & 15,1 & 15,0 & 12,8 \\
\hline
\end{tabular}

CUADRO 2

MASA DE $130 \mathrm{kp} / \mathrm{cm}^{2}$

(Resistencia a la compresión en $\mathrm{kp} / \mathrm{cm}^{2}$ )

\begin{tabular}{|c|c|c|c|c|}
\hline \multirow{2}{*}{$\begin{array}{l}\text { Masa } \\
\text { núm. }\end{array}$} & \multicolumn{2}{|c|}{$\begin{array}{l}\text { Pillares moldeados } \\
25 \times 25 \mathrm{~cm} \\
\mathrm{~h}=2 \mathrm{~m}\end{array}$} & \multicolumn{2}{|c|}{$\begin{array}{l}\text { Probetas cilíndricas moldeadas } \\
\qquad \begin{array}{c}3=15 \mathrm{~cm} \\
\mathrm{~h}=30 \mathrm{~cm}\end{array}\end{array}$} \\
\hline & $\underset{\text { Sinn }}{\text { perforar }}$ & $\begin{array}{l}\text { Perforado y } \\
\text { relleno }\end{array}$ & $\begin{array}{l}\text { Conservadas al aire } \\
20 \pm 1^{\circ} \mathrm{C}>80 \% \mathrm{HR}\end{array}$ & $\begin{array}{c}\text { Conservadas en agua } \\
20 \pm 1^{\circ} \mathrm{C}\end{array}$ \\
\hline 1 & 128,5 & 120,0 & $\begin{array}{r}133,0-124,5-106,5 \\
129,5-91,0-117,2\end{array}$ & $\begin{array}{r}134,1-129,0-110,5 \\
103,5-90,5-129,0\end{array}$ \\
\hline 2 & 137,0 & 140,0 & $\begin{array}{l}123,5-135,5-103,5 \\
111,0-110,0-106,0\end{array}$ & $\begin{array}{l}132,0-135,5-106,5 \\
126,0-113,0-107,5\end{array}$ \\
\hline 3 & 135,5 & 132,5 & $\begin{array}{l}133,0-133,5-108,5 \\
132,0-115,0-105,0\end{array}$ & $\begin{array}{r}143,5-120,0-104,5 \\
121,5-110,5-98,0\end{array}$ \\
\hline 4 & 120,0 & 127,0 & $\begin{array}{r}114,5-102,0-106,5 \\
106,0-77,0-68,2\end{array}$ & $\begin{array}{l}140,0-99,0-105,5 \\
127,5-97,0-105,0\end{array}$ \\
\hline 5 & 130,0 & 109,5 & $\begin{array}{l}132,0-124,0-124,5 \\
121,0-133,5-126,5\end{array}$ & $\begin{array}{l}137,0-131,0-147,0 \\
133,0-125,5-131,5\end{array}$ \\
\hline 6 & 116,5 & 133,5 & $\begin{array}{l}129,0-135,5-117,0 \\
154,5-127,5-124,0\end{array}$ & $\begin{array}{l}152,0-145,5-146,0 \\
120,0-150,0-127,5\end{array}$ \\
\hline $\begin{array}{l}\text { Valor } \\
\text { medio }\end{array}$ & 127,9 & 127,1 & 117,8 & 123,2 \\
\hline $\begin{array}{c}\text { Coef. var. } \\
(\%)\end{array}$ & 6,4 & 8,6 & 14,5 & 13,7 \\
\hline
\end{tabular}


CUADRO 3

MASA DE $90 \mathrm{kp} / \mathrm{cm}^{2}$

[Resistencia a la compresión en $\mathrm{kp} / \mathrm{cm}^{2}$ ]

Testigos cilíndricos cortados a diferentes alturas $(H)$ sobre la base del pilar, $h=10 \mathrm{~cm}, \mathrm{~h}=20 \mathrm{~cm}$ Rotura en seco y saturados

\begin{tabular}{|c|c|c|c|c|c|c|c|c|c|}
\hline \multirow{2}{*}{$\underset{(\mathrm{cm})}{\text { Altura }} \mathbf{H}$} & \multirow{2}{*}{ Estado } & \multicolumn{6}{|c|}{ Masa número } & \multirow{2}{*}{$\begin{array}{l}\text { Valor } \\
\text { medio }\end{array}$} & \multirow{2}{*}{$\begin{array}{c}\text { Coeficiente } \\
\text { var. } \%\end{array}$} \\
\hline & & 1 & 2 & 3 & 4 & 5 & 6 & & \\
\hline 15 & Seco & 158,7 & 149,4 & 158,6 & 159,6 & 151,9 & 200,2 & 163,1 & 15,0 \\
\hline \multirow{2}{*}{30} & Seco & 124,5 & 189.5 & 144,5 & 167,5 & 177,0 & 136,0 & 156,5 & 16,2 \\
\hline & Sat. & 88,5 & 140,0 & 108,0 & 169,5 & 164,0 & 110,0 & 130,0 & $2.5,3$ \\
\hline \multirow{2}{*}{58} & Seco & 910 & 110,0 & 173,0 & 118,0 & 124,5 & 152,0 & 128.1 & 23,2 \\
\hline & Sat. & 92,0 & 110,0 & 97,0 & 97,0 & 118,5 & 104,0 & 103,1 & 9,5 \\
\hline \multirow{2}{*}{86} & Seco & 86,5 & 124,0 & 101,0 & 123,0 & 102.0 & 120,0 & 109,4 & 13,9 \\
\hline & Sat. & 39,0 & 110,0 & 95,5 & 92,5 & 91,8 & 76,0 & 92,4 & 11,8 \\
\hline \multirow{2}{*}{114} & Seco & 114,5 & 126,5 & 104,0 & 135,0 & 71,5 & 130,5 & 113,7 & 20,7 \\
\hline & Sat. & 111,0 & 90,0 & 81,0 & 127,0 & 63,2 & 94,0 & 94,3 & 23,7 \\
\hline \multirow{2}{*}{142} & Seco & 103.0 & 114,5 & 94,0 & 112,0 & 93,7 & 116,0 & 105,5 & 9,6 \\
\hline & Sat. & 85,0 & 126,0 & 77,0 & 110,0 & 79,3 & 94,0 & 95,2 & 20,2 \\
\hline \multirow{2}{*}{170} & Seco & 70,0 & 47,5 & 101,0 & 65,0 & 77,8 & 91,0 & 75.4 & 25,3 \\
\hline & Sat. & 90,0 & 42,0 & 88,5 & 50,5 & 83,0 & 68,0 & 70,3 & 29,0 \\
\hline 185 & Seco & 93,4 & 56,8 & 94,1 & 60,1 & 99,3 & 91,5 & 82,6 & 24,4 \\
\hline
\end{tabular}

\section{CUADRO 4}

MASA DE $130 \mathrm{kp} / \mathrm{cm}^{2}$

(Resistencia a la compresión en $\mathrm{kp} / \mathrm{cm}^{2}$ ]

\begin{tabular}{|c|c|c|c|c|c|c|c|c|c|}
\hline \multicolumn{10}{|c|}{$\begin{array}{c}\text { Testigos cilíndricos cortados a diferentes alturas }(H) \text { sobre la base del pílar, } c=10 \mathrm{~cm}, \mathrm{~h}=20 \mathrm{~cm} \\
\text { Rotura en seco y saturados }\end{array}$} \\
\hline \multirow{2}{*}{$\begin{array}{l}\text { Altura } H \\
(\mathrm{~cm})\end{array}$} & \multirow{2}{*}{ Estado } & \multicolumn{6}{|c|}{ Masa número } & \multirow{2}{*}{$\begin{array}{l}\text { Valor } \\
\text { medio }\end{array}$} & \multirow{2}{*}{$\begin{array}{l}\text { Coeficiente } \\
\text { var. } \%\end{array}$} \\
\hline & & 1 & 2 & 3 & 4 & 5 & 6 & & \\
\hline 15 & Seco & 223,0 & 219,8 & 240,6 & 209,1 & 227,6 & 265,0 & 230,8 & 13,0 \\
\hline \multirow{2}{*}{30} & Seco & 211,5 & 228,0 & 191,0 & 173,5 & 179,0 & 207,0 & 198.3 & 10,5 \\
\hline & Sat. & 178,5 & 187,5 & 162,0 & 134,0 & 179,5 & 161,0 & 167,1 & 11,5 \\
\hline \multirow{2}{*}{58} & Seco & 177,5 & 195,5 & 205,0 & 179,0 & 169,5 & 182,5 & 184,8 & 7,0 \\
\hline & Sat. & 154,0 & 180,0 & 128,0 & 146,0 & 159,5 & 160,5 & 154,7 & 11,1 \\
\hline \multirow{2}{*}{86} & Seco & 157,0 & 190,5 & 173,0 & 133,5 & 161,0 & 189,5 & 167,4 & 12,9 \\
\hline & Sat. & 139,5 & 160,0 & 137,0 & 153,0 & 157,5 & 173,0 & 153,3 & 8,8 \\
\hline \multirow{2}{*}{114} & Seco & 167,0 & 163,5 & 176,0 & 177,5 & 151,0 & 190,0 & 170,8 & 7,8 \\
\hline & Sat. & 142,0 & 136,0 & 108,0 & 131,5 & 152,5 & 159,5 & 138,2 & 13,1 \\
\hline \multirow{2}{*}{142} & Seco & 120,0 & 161,0 & 161,0 & 154,5 & 172,5 & 178,0 & 157,8 & 12,9 \\
\hline & Sat. & 103,5 & 137,0 & 128,5 & 113,0 & 153,0 & 147,5 & 130,4 & 14,8 \\
\hline \multirow{2}{*}{170} & Seco & 148,0 & 169,0 & 153,0 & 134,5 & 156,5 & 148,0 & 151,5 & 7,5 \\
\hline & Sat. & 135,5 & 129,0 & 134,0 & 103,5 & 130,5 & 142,5 & 129,2 & 10,4 \\
\hline 185 & Seco & 142,7 & 151,0 & 138,7 & 129,8 & 169,4 & 172,6 & 150,7 & 14,4 \\
\hline
\end{tabular}




\section{CONCLUSIONES}

\subsection{Representatividad de las probetas moldeadas}

La comparación de la resistencia de pilares de tamaño natural $(25 \times 25 \times 200 \mathrm{~cm})$ con la de probetas cilíndricas normalizadas $(15 \times$ $\times 30 \mathrm{~cm}$ ), confeccionadas con el mismo hormigón de los pilares, la hemos efectuado a partir de los cuadros de resultados 1 y 2 . Expresamos el resumen en el cuadro 5.

No cabe duda, a la vista de las cifras, que las probetas cilíndricas moldeadas son una buena representación de la resistencia del elemento de obra a que pertenecen. La resistencia de obra resulta incluso algo superior.

La conclusión resulta lógica si se tiene en cuenta que las roturas de los pilares se han producido casi siempre por su parte superior, cuyo hormigón ha sido sometido a menor presión durante la puesta en obra y compactación, de forma parecida a la de las probetas que son de pequeña altura (foto 1).

Por otra parte no se observa una diferencia significativa entre los resultados de las probetas conservadas en un ambiente de humedad no excesivamente riguroso $(>80 \%)$ y las conservadas en agua rotas saturadas.

Ocurrirá probablemente que la mejora esperable de resistencia en las probetas curadas en agua respecto a las conservadas al aire se compensará con la disminución de resistencia que se produce por romperlas saturadas (ver apartado 2.4).

\subsection{Variación de la resistencia del pilar con la altura}

Para estudiar esta variación y la consiguiente representatividad de los testigos cortados a diferentes alturas respecto a la resistencia

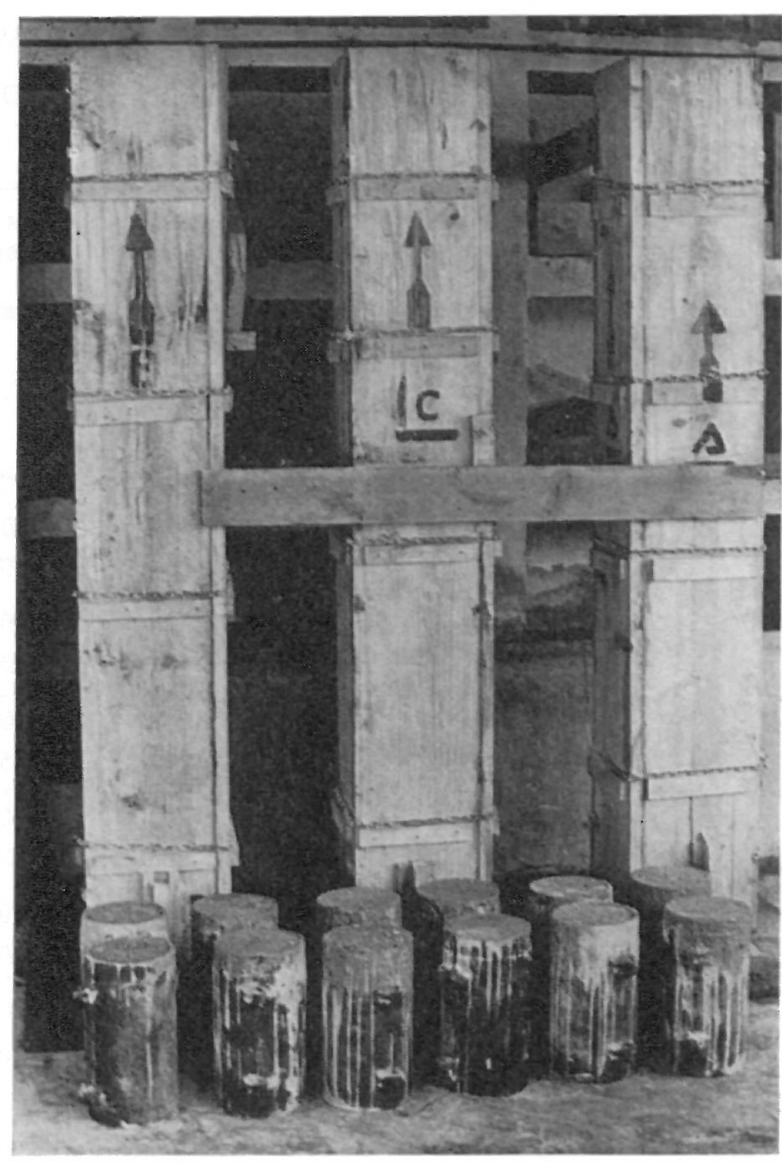

Foto 1. Preparación de pilares de $25 \times 25 \times 200 \mathrm{~cm}$ y de probetas cilíndricas normalizadas.

del pilar, se han preparado dos series de pilares de $25 \times 25 \times 200 \mathrm{~cm}$ en calidades de hormigón 90 y $130 \mathrm{kp} / \mathrm{cm}^{2}$.

De cada serie, la mitad de los pilares se han roto normalmente, y de la otra mitad se han cortado testigos de $10 \mathrm{~cm}$ de diámetro, a diferentes alturas sobre su base, que se han ensayado a compresión, un grupo saturados por inmersión 48 horas en agua, de acuerdo con la norma UNE 7241 , y otra en seco conservados en el ambiente del laboratorio.

\section{CUADRO 5}

Edad: 150 días

\begin{tabular}{|c|c|c|c|}
\hline \multirow{2}{*}{ ELEMENTO } & \multirow{2}{*}{ Curado } & \multicolumn{2}{|c|}{ Calidad teórica } \\
\hline & & $90 \mathrm{kp} / \mathrm{cm}^{2}$ & $130 \mathrm{kp} / \mathrm{cm}^{2}$ \\
\hline Pilar de $25 \times 25 \times 200 \mathrm{~cm} \ldots \ldots$ & Aire & $82,4(100 \%)$ & $127,9(100 \%)$ \\
\hline Probeta de $15 \times 30 \mathrm{~cm} \ldots \ldots \ldots$ & Aire & $77,0 \quad(93 \%)$ & $117,8 \quad(92 \%)$ \\
\hline Probeta de $15 \times 30 \mathrm{~cm} \ldots \ldots \ldots$ & Agua & $79,2 \quad(96 \%)$ & $123,2 \quad(96 \%)$ \\
\hline
\end{tabular}


Los resultados de todos estos ensayos aparecen en los cuadros 3 y 4 , y a partir de ellos se han representado en las figuras 2 y 3 los valores medios de las resistencias de los testigos, junto con algunas relaciones derivadas de los mismos, referidos a su altura respecto a la base del pilar.

Los testigos se cortaron siempre perpendicularmente al eje del pilar, salvo los obtenidos

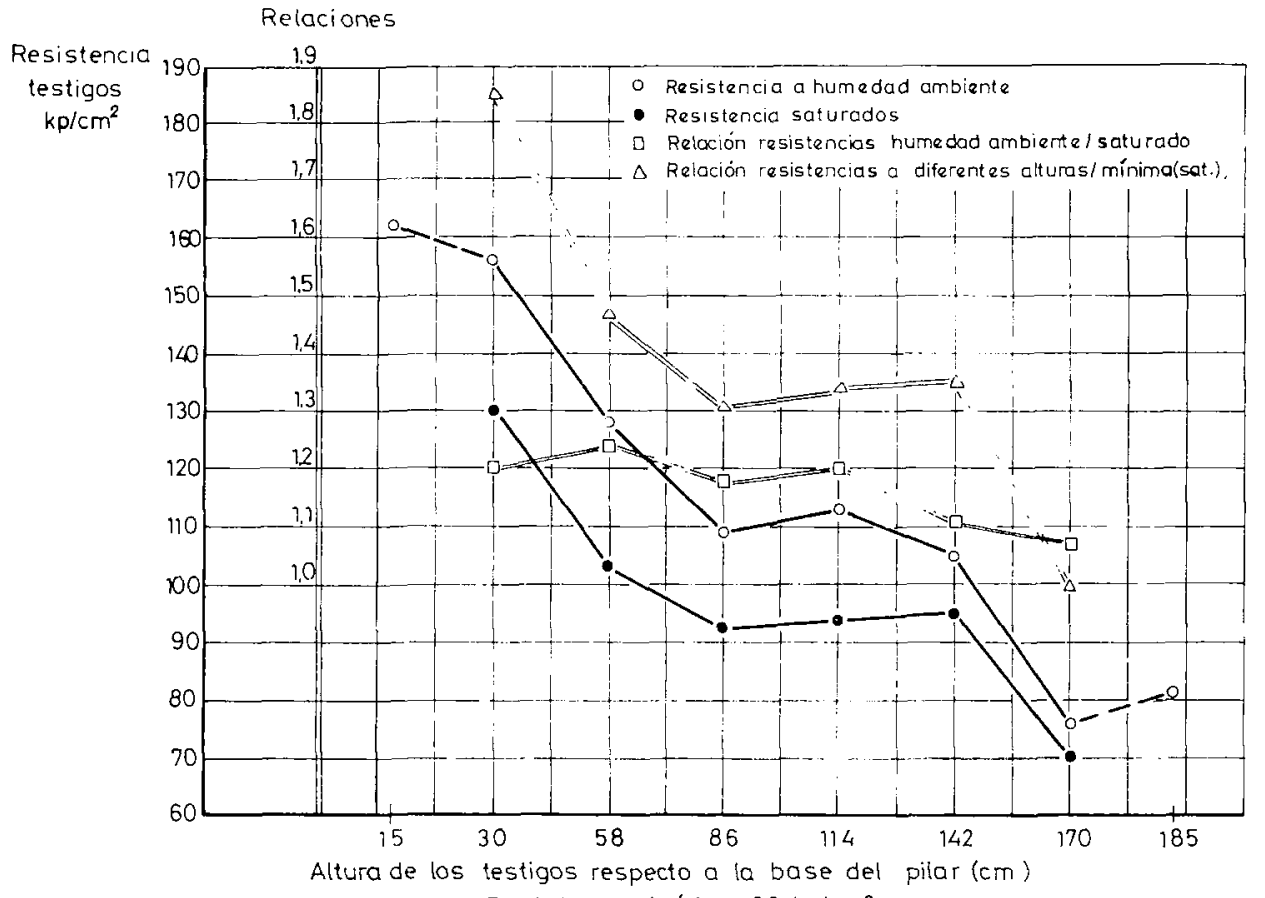

Fig. 2 Resistencia teórica $90 \mathrm{kp} / \mathrm{cm}^{2}$

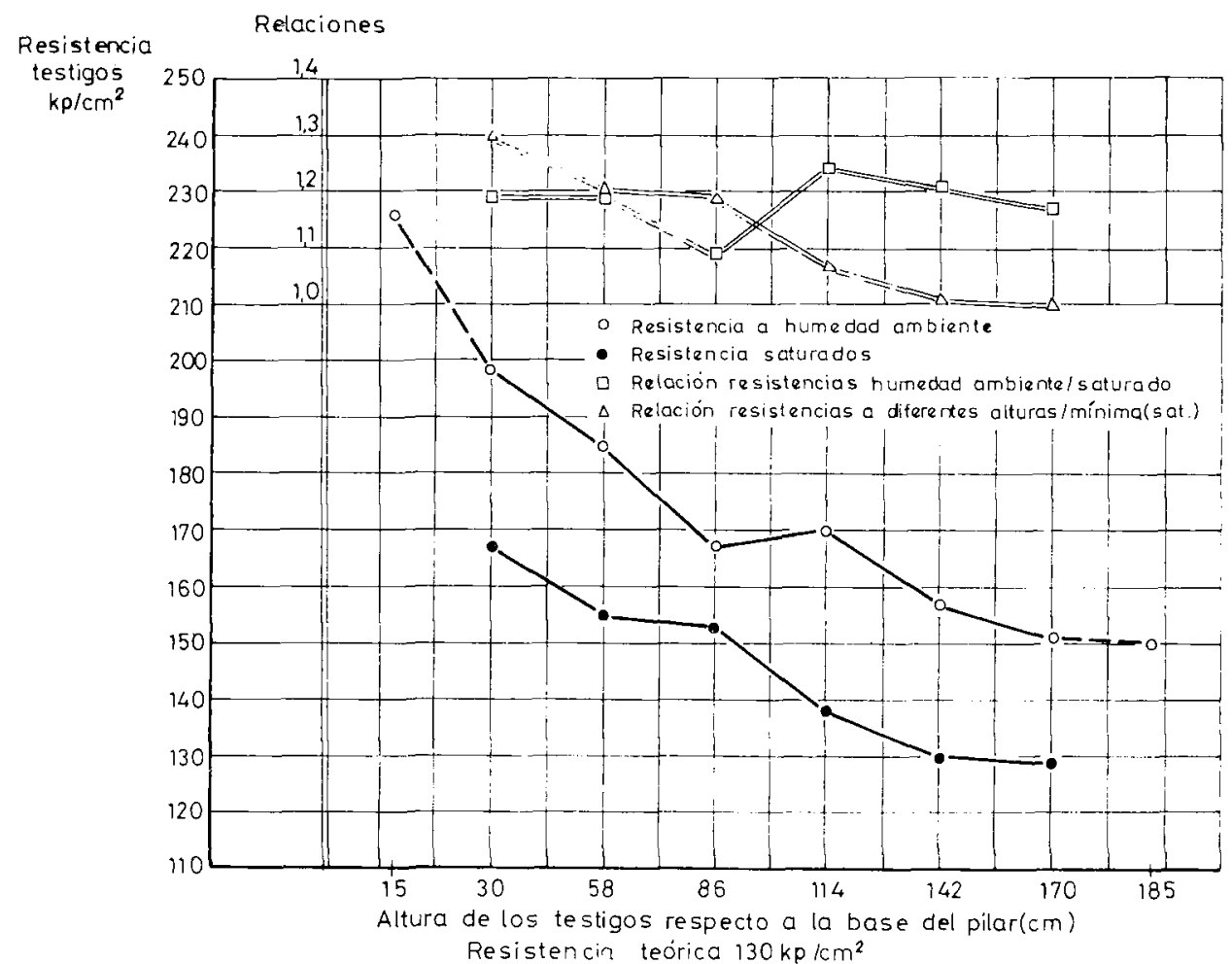

Fig. 3 
a 15 y $185 \mathrm{~cm}$, que se obtuvieron paralelamente al eje, representados en las figuras 2 y 3 por puntos unidos por líneas de trazos discontinuos a las curvas de resistencia.

A la vista de los gráficos se hace notar la gran diferencia de resistencia existente entre la parte superior e inferior del pilar. Este fenómeno, ya conocido, se debe a la mayor compactación que resulta en la zona inferior de un pilar por el peso de las capas superiores, y la peor calidad de estas últimas por la segregación del árido grueso y la acumulación de agua y arena.

Nuestra aportación en relación a este hecho es la puesta de manifiesto con las cifras correspondientes del aumento de esta diferencia y la consiguiente inseguridad, a medida que la calidad del hormigón es más baja.

El examen del cuadro 6 nos permite comprobar que en un pilar de edificación, hecho con una calidad nominal de hormigón de $130 \mathrm{kp}$ por $\mathrm{cm}^{2}$, el hormigón de la parte superior puede tener una resistencia menor del $70 \%$ de la correspondiente a la parte inferior. $Y$ lo que es más alarmante, que en un pilar ya de muy baja calidad nominal, $90 \mathrm{kp} / \mathrm{cm}^{2}$, y por tanto no admisible por Normas, la resistencia de la parte superior puede ser menor de la mitad de la inferior.

Esto nos lleva a ponderar la importancia que tiene el punto de extracción de los testigos en la inspección de obras ya terminadas. Realmente la probeta se debería sacar de la zona superior, que es la de menor resistencia, puesto que el pilar fallará por el punto más. débil.

Sin embargo, como ello puede ser difícil, lo que es al menos fundamental es valorar por un medio no destructivo la resistencia del pilar a diferentes alturas, incluyendo la superior, y tarar este aparato no destructivo con probetas testigo cortadas de zonas que nos convengan.

\subsection{Representatividad de los testigos corta- dos respecto a la resistencia del pilar}

Para poner de manifiesto lo dicho al final del apartado anterior se han agrupado en el cuadro 7 los resultados del ensayo a compresión de pilares a tamaño natural, de las probetas moldeadas correspondientes y de testigos extraídos a tres alturas respecto a la base: de la parte inferior del pilar, de la superior y de $70 \mathrm{~cm}$ sobre la base, que es una altura usual de extracción de probetas con trépano, debido a las medidas del aparato extractor (fotos 2 y 3 ).

Todos los valores dados corresponden, para mejor comparación, a roturas hechas a humedad ambiente de laboratorio, dado que los pilares no se rompieron en estado de saturación de agua.

\section{CUADRO 6}

RELACION ENTRE RESISTENCIAS DE TESTIGOS DE $10 \mathrm{~cm}$ DE DIAMETRO CORTADOS A DIFERENTES ALTURAS SOBRE LA BASE DE UN PILAR DE $2 \mathrm{~m}$ DE ALTURA, RESPECTO A LA RESISTENCIA DE LA PARTE SUPERIOR

\begin{tabular}{|c|c|c|c|c|}
\hline \multirow{3}{*}{$\begin{array}{c}\text { Altura sobre la base } \\
\text { del pilar } \\
(\mathrm{cm})\end{array}$} & \multicolumn{4}{|c|}{ Calidad teórica de hormigón } \\
\hline & \multicolumn{2}{|c|}{$90 \mathrm{kp} / \mathrm{cm}^{2}$} & \multicolumn{2}{|c|}{$130 \mathrm{kp} / \mathrm{cm}^{2}$} \\
\hline & $\begin{array}{l}\text { Humedad } \\
\text { ambiente }\end{array}$ & Saturados & $\begin{array}{l}\text { Humedad } \\
\text { ambiente }\end{array}$ & Saturados \\
\hline 185 & 1,10 & - & 0,99 & - \\
\hline 170 & 1,00 & 1,00 & 1,00 & 1,00 \\
\hline 142 & 1,40 & 1,35 & 1,04 & 1,01 \\
\hline 114 & 1,51 & 1,34 & 1,13 & 1,07 \\
\hline 86 & 1,45 & 1,31 & 1,10 & 1,19 \\
\hline 58 & 1,70 & 1,47 & 1,22 & 1,20 \\
\hline 30 & 2,08 & 1,85 & 1,31 & 1,30 \\
\hline 15 & 2,16 & - & 1,51 & - \\
\hline
\end{tabular}




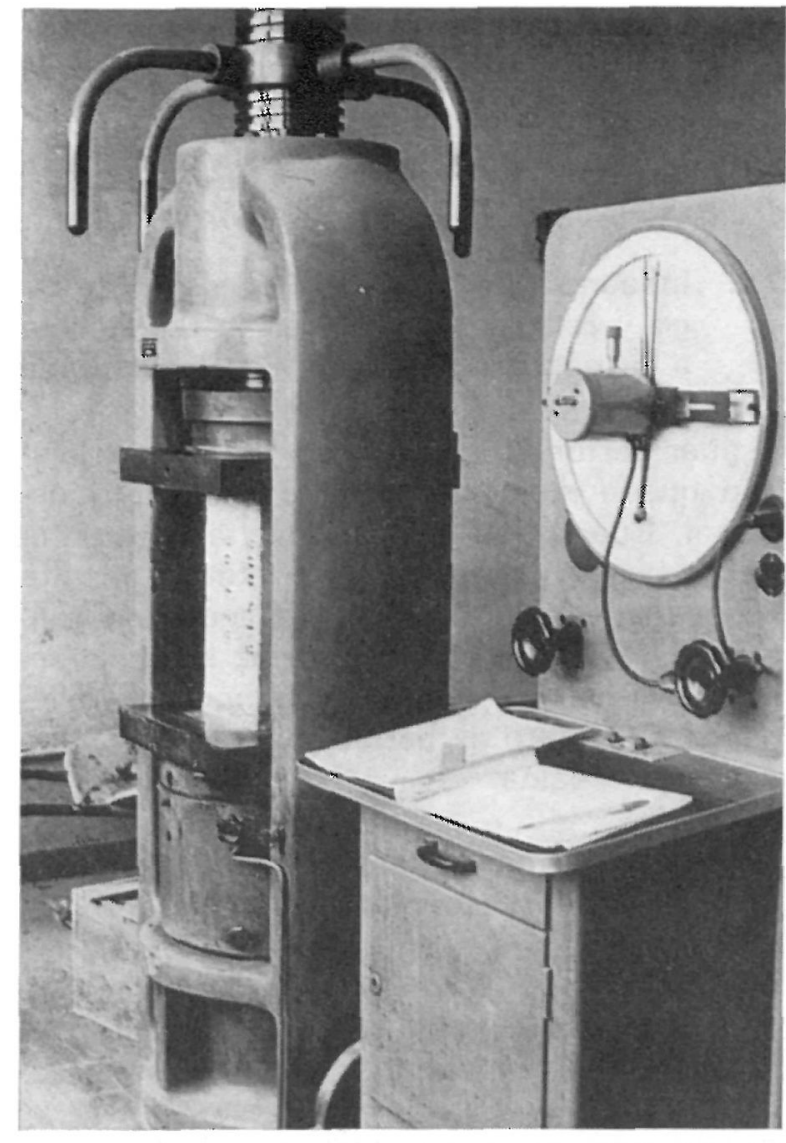

Foto 2. Prensa Amsler para ensayo de probetas y testigos.

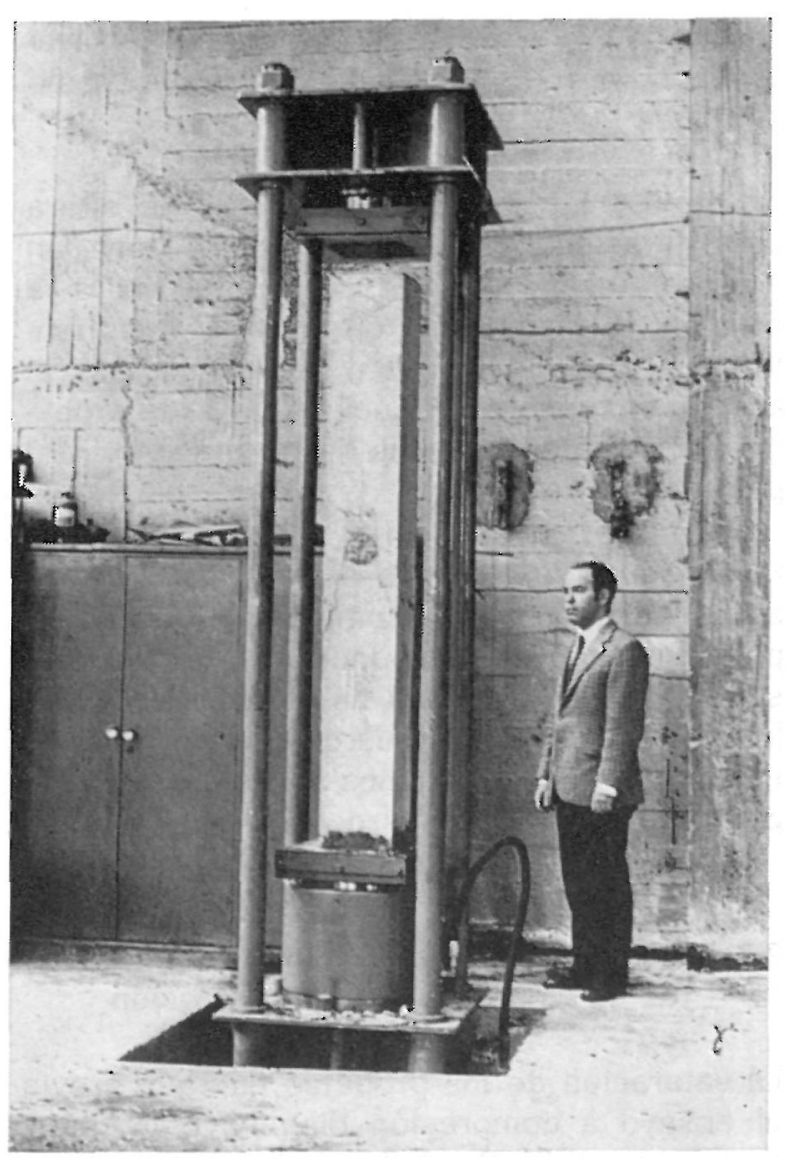

Foto 3. Prensa construida para la rotura de pilares.

\section{CUADRO 7}

\begin{tabular}{|c|c|c|c|c|c|c|}
\hline \multirow{2}{*}{$\begin{array}{c}\text { Calidad } \\
\text { teórica } \\
\text { del hormigón }\end{array}$} & \multirow{2}{*}{ Valores determinados } & \multirow{2}{*}{$\begin{array}{c}\text { Pilares } \\
\square 25 \times 25 \mathrm{~cm} \\
\mathrm{~h}=2 \mathrm{~m}\end{array}$} & \multicolumn{3}{|c|}{$\begin{array}{c}\text { Testigos cortados } \phi=10 \mathrm{~cm} \\
\text { Altura respecto a la base }\end{array}$} & \multirow{2}{*}{$\begin{array}{c}\text { Probetas } \\
\text { moldeadas } \\
\phi=15 \mathrm{~cm} \\
\mathrm{~h}=30 \mathrm{~cm}\end{array}$} \\
\hline & & & $15 \mathrm{~cm}$ & $70 \mathrm{~cm}$ & $185 \mathrm{~cm}$ & \\
\hline \multirow{3}{*}{$90 \mathrm{kp} / \mathrm{cm}^{2}$} & $\begin{array}{l}\text { Resistencia media } \\
\quad\left(\mathrm{kp} / \mathrm{cm}^{2}\right)\end{array}$ & $\begin{array}{c}82,4 \\
(100 \%)\end{array}$ & $\begin{array}{c}163,1 \\
(198 \%)\end{array}$ & $\begin{array}{c}116,0 \\
(141 \%)\end{array}$ & $\begin{array}{c}82,6 \\
(100 \%)\end{array}$ & $\begin{array}{c}77,0 \\
(93 \%)\end{array}$ \\
\hline & $\begin{aligned} \text { Coeficiente } & \text { de } \\
\text { variación } & (\%)\end{aligned}$ & 7,9 & 15,0 & 13,9 & 24,4 & 15,0 \\
\hline & $\begin{array}{l}\text { Valor mínimo } \\
\qquad\left(\mathrm{kp} / \mathrm{cm}^{2}\right)\end{array}$ & 72,5 & 129,0 & - & 47,5 & 61,0 \\
\hline \multirow{3}{*}{$130 \mathrm{kp} / \mathrm{cm}^{2}$} & $\begin{array}{l}\text { Resistencia media } \\
\left(\mathrm{kp} / \mathrm{cm}^{2}\right)\end{array}$ & $\begin{array}{c}127,9 \\
(100 \%)\end{array}$ & $\begin{array}{c}229,4 \\
(179 \%)\end{array}$ & $\begin{array}{c}173,0 \\
(135 \%)\end{array}$ & $\begin{array}{c}150,7 \\
(118 \%)\end{array}$ & $\begin{array}{c}117,8 \\
(92 \%)\end{array}$ \\
\hline & $\begin{array}{cc}\text { Coeficiente } & \text { de } \\
\text { variación } & (\%)\end{array}$ & 6,4 & 13,0 & 12,9 & 14,4 & 14,5 \\
\hline & $\begin{array}{l}\text { Valor mínimo } \\
\left(\mathrm{kp} / \mathrm{cm}^{2}\right)\end{array}$ & 116,5 & 185,0 & - & 101,0 & 68,2 \\
\hline
\end{tabular}

Examinando los porcentajes que suponen cada resistencia respecto a la de los pilares, vemos la bastante buena representación de la resistencia del pilar por los testigos extraídos de la parte superior. En el caso de la calidad in- ferior $\left(90 \mathrm{kp} / \mathrm{cm}^{2}\right)$ hay coincidencia de valores, y en las otras $\left(130 \mathrm{kp} / \mathrm{cm}^{2}\right)$ los testigos dan una resistencia algo más optimista, $+18 \%$, que la de los pilares correspondientes. Son lógicos estos resultados teniendo en 
cuenta que la mayoría de los pilares ensayados han roto en la proximidad del extremo superior.

En cambio los testigos cortados a la altura normal de trabajo del trépano, $70 \mathrm{~cm}$, dan una resistencia del 35 al $40 \%$ superior a la del pilar. Se cometería un error ya importante desde el punto de vista de la seguridad, al aceptar los valores deducidos de esas probetas como representativos de la resistencia del pilar.

Finalmente, si se cortaran las probetas de zonas especialmente compactadas como la parte inferior del pilar, podríamos obtener resistencias del orden del doble que las reales, lo que, en ausencia de un conocimiento cuantitativo de los fenómenos que comentamos, podría encerrar verdadero peligro.

\subsection{Influencia de la saturación de las probe- tas en la resistencia a compresión}

La saturación de las probetas en agua previa al ensayo a compresión disminuye la resistencia obtenida. Se deduce claramente de la comparación de resultados de los testigos cilíndricos de $10 \times 20 \mathrm{~cm}$ sacados por parejas a diferentes alturas sobre la base del pilar y rotos uno a humedad ambiente de laboratorio y otro saturado.

Examinando en las figuras 2 y 3 las curvas que dan los valores de los cocientes, resistencia ambiente laboratorio/saturados se observa una cierta regularidad de sus valores en relación con el nivel de resistencia del testigo. Los valores medios de estos cocientes son para el hormigón de $90 \mathrm{kp} / \mathrm{cm}^{2}$ de resistencia teórica, 1,17, y para el de $130 \mathrm{kp}$ por $\mathrm{cm}^{2}, 1,18$.

Se puede concluir, por tanto, en el intervalo de resistencias manejado $\left(70\right.$ a $200 \mathrm{kp} / \mathrm{cm}^{2}$ ) que al romper los testigos conservados después de su extracción en el ambiente normal de laboratorio aumenta el valor de su resistencia en un $17-18 \%$ respecto a la de los mismos testigos saturados de agua previamente a su rotura. De ahí la importancia real de observar la condición de saturación, que a menudo se soslaya, si se quiere cumplir con las normas de ensayo que así lo especifican (UNE 7241 ).

Quizás sea discutible si no se podría en algunos casos tomar como resistencia a conside- rar para la obra la de los testigos ensayados en seco, dado que en muchas zonas de la obra es difícil que se den en la práctica las condiciones de saturación.

\subsection{Influencia de la extracción de un testi- go y relleno posterior en la resistencia a la compresión de la pieza perforada}

El pilar perforado por la extracción de un testigo queda disminuido en su sección transversal. Cuando su calidad resulta suficientemente buena y no precisa de refuerzo resulta necesario evitar el efecto del agujero y restituirle de la manera más eficaz su resistencia primitiva. En general esto se hace rellenando el agujero de hormigón con alguna adición o de resina.

Uno de nuestros propósitos ha sido también estudiar el comportamiento resistente de estos pilares a los que después de extraerles una probeta de $10 \mathrm{~cm}$ de diámetro se rellena de diversas formas el agujero provocado para la extracción del testigo.

En una primera fase se hicieron pruebas comparativas con prismas de $25 \times 25 \times 50 \mathrm{~cm}$ a los que se perforaba el agujero, para estudiar el método más eficaz de relleno. Se utilizaron para éste masas de hormigón de varias consistencias y dosificación, adiciones de expansivo e incluso se estudió la eficacia de aplicar a las paredes del agujero resina epoxi para mejorar la adherencia del relleno (INFORMES DE LA CONSTRUCCION, núm. 255, noviembre 1973).

Como resultado de estas pruebas previas se vio la conveniencia de emplear para el relleno una masa de hormigón de resistencia elevada, con la adición de un ligero expansivo, y de consistencia blanda que garantizase un total relleno del agujero. No se observó en cambio mejora con la aplicación previa de resina epoxi a sus paredes, por lo que en el estudio final se ha prescindido de ésta.

Las pruebas definitivas se han realizado sobre 12 parejas de pilares de armadura despreciable en dos calidades de hormigón, 90 y $130 \mathrm{kp} / \mathrm{cm}^{2}$. Sus dimensiones eran $2 \mathrm{~m}$ de altura, con una sección pequeña, $25 \times 25 \mathrm{~cm}$, para que fueran afectados en mayor grado por la extracción de un testigo de $10 \mathrm{~cm}$ de diámetro. La perforación se realizaba de cara a cara del pilar, afectando, pues, el corte a un $40 \%$ de su sección transversal (fotos 3 y 4 ) 


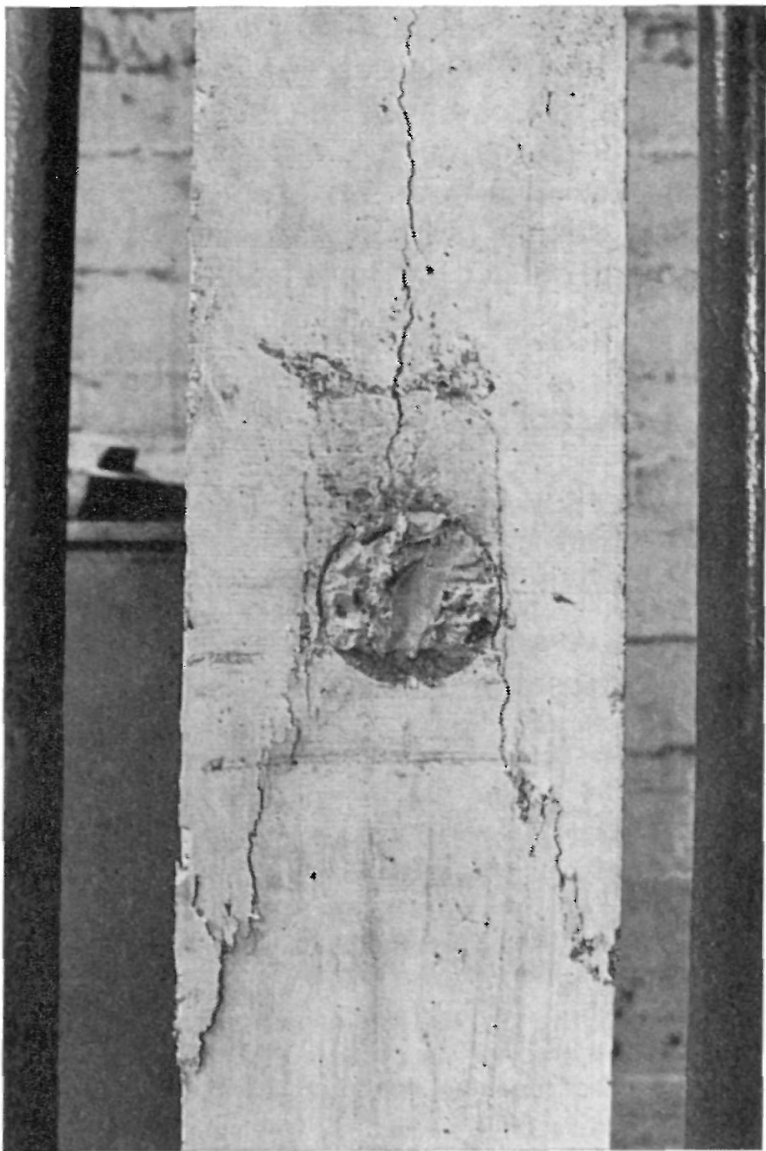

Foto 4. Rotura de pilar por la zona central (caso singular).

Los ensayos se realizaron a la edad de 150 días, teniendo en ese momento el relleno 90 días, y $250 \mathrm{kp} / \mathrm{cm}^{2}$, estando los resultados reflejados en el cuadro 8 .

El análisis de los grados de eficacia del relleno, entendiéndose por ello el porcentaje de resistencia alcanzado por la pieza rellena en relación con la no perforada, revela, desde el punto de vista de los valores medios, unos resultados muy satisfactorios: 90 y $99 \%$.

La consideración de los valores mínimos, sobre todo el correspondiente a la peor calidad del hormigón, $69 \%$, podría preocupar debido a suponer una apreciable reducción de resistencia. Sin embargo, hay dos consideraciones que estimamos pueden alejar dicho temor.

La mayor parte de los pilares han roto por la parte superior, que ha fallado por su menor resistencia que la zona del centro del pilar en que estaba situado el agujero rellenado. Las dispersiones de resistencias son, por lo tanto, achacables en una gran parte a la propia del hormigón de la parte superior y no al efecto del agujero y posterior relleno.

En segundo lugar, y contemplando el efecto anterior desde otro punto de vista, cuando se extraigan los testigos de la parte inferior del pilar podremos estar tranquilos de que, aunque haya una pequeña disminución de resiscia en la sección de extracción del testigo, sin embargo será ésta mayor que la de la zona superior del pilar.

Con todo estimamos que rellenando el agujero causado por el trépano con un hormigón de alta calidad, con un ligero expansivo, parecen esperables pérdidas de resistencia menores del $15 \%$. Claro está que todas las cifras manejadas son para las dimensiones transversales del testigo $(10 \mathrm{~cm})$ y pilar $(25 \times 25 \mathrm{~cm})$ utilizadas en estos ensayos, cuya combinación es muy desfavorable. Para tamaños mayores del pilar su pérdida de resistencia será menor, en relación inversa con dicho tamaño. Además, finalmente, queda sin afectarse la capacidad resistente de las armaduras, lo que supone que la pequeña disminución de la resistencia de la sección de hormigón influye aún en menor proporción en la resistencia global del pilar.

\section{RESUMEN}

Siempre dentro del marco de las investigaciones realizadas, podemos resumir de todo lo anterior las siguientes conclusiones:

\section{CUADRO 8}

\begin{tabular}{|c|c|c|c|c|c|}
\hline \multirow{2}{*}{$\begin{array}{l}\text { Calidad teórica } \\
\text { del hormigón }\end{array}$} & \multicolumn{2}{|c|}{$\begin{array}{c}\text { Resistencia media } \\
\text { de pilares de } 25 \times 25 \times 200 \mathrm{~cm} \\
\left(\mathrm{kp} / \mathrm{cm}^{2}\right)\end{array}$} & \multicolumn{3}{|c|}{$\begin{array}{l}\text { Grado de eficacía del relleno } \\
(\%)\end{array}$} \\
\hline & Sin perforar & $\begin{array}{l}\text { Perforación } \\
\text { de } 10 \mathrm{~cm} \varnothing \\
\text { y relleno }\end{array}$ & $\begin{array}{l}\text { Valor } \\
\text { medio }\end{array}$ & $\begin{array}{l}\text { Valor } \\
\text { mínimo }\end{array}$ & Recorrido \\
\hline $90 \mathrm{kp} / \mathrm{cm}^{2}$ & 82,4 & 74,0 & 90 & 69 & 35 \\
\hline $130 \mathrm{kp} / \mathrm{cm}^{2}$ & 127,9 & 127,1 & 99 & 84 & 30 \\
\hline
\end{tabular}


a) Las probetas cilíndricas moldeadas son una buena representación de la resistencia del elemento de obra a que pertenecen. La resistencia de obra puede ser incluso de un 5 a un $10 \%$ superior.

b) En pilares de un hormigón de la menor calidad admitida en Normas $\left(130 \mathrm{kp} / \mathrm{cm}^{2}\right)$, el hormigón de la parte superior puede tener una resistencia menor del $70 \%$ de la correspondiente a la parte inferior. Cuando el hormigón es de una calidad muy baja (90 kp/ $\mathrm{cm}^{2}$, por ejemplo), la resistencia de la parte inferior será más del doble que la de la superior.

c) Los testigos cortados a la altura normal de trabajo del trépano $(70 \mathrm{~cm})$ dan una resistencia del 35 al $40 \%$ superior a la del pilar de que se han extraído.

d) El romper los testigos conservados a humedad ambiente de laboratorio en vez de saturados en agua, como indica la Norma UNE 7241 , supone un aumento de su resistencia del 17 al $18 \%$.

e) Un método suficientemente eficaz de relleno del agujero causado en un pilar por la extracción de un testigo cilíndrico puede ser el emplear hormigón de alta calidad, en consistencia blanda, con la adi. ción de un ligero expansivo. Las pérdidas esperables de resistencia de la sección de hormigón en un pilar que queda en estas condiciones, estimamos serán menores de un $15 \%$, dependiendo de la relación entre su sección transversal y tamaño de testigo. Al no afectarse la capacidad resistente de las armaduras la resistencia global del pilar quedará todavía menos disminuida.

Finalmente, para completar este resumen de conclusiones, añadimos a las anteriores dos procedentes de nuestro trabajo anterior (INFORMES DE LA CONSTRUCCION, núm. 255, 1973).

f) Los testigos de 10 y $15 \mathrm{~cm}$ de diámetro extraídos con trépano de hormigones de tamaño máximo de árido $2,5-3 \mathrm{~cm}$ presentan un coeficiente de variación de su resistencia comprendido entre el 5 y el $10 \%$, valor muy pequeño y análogo al de las probetas cilíndricas moldeadas. Resultan, por lo tanto, ser datos con alto grado de confianza.

No así los testigos de $5 \mathrm{~cm}$ de diámetro, cuyo coeficiente de variación sube hasta 15 a $30 \%$.

g) El deterioro de la resistencia de los testigos por efecto del corte, en hormigo- nes de tamaño máximo de árido $2,5-3 \mathrm{~cm}$ es, suponiéndolo despreciable en los testigos de $15 \mathrm{~cm}$ de diámetro, de un 0,5 $4,2 \%$ en los de $10 \mathrm{~cm}$ de diámetro, y de un 14,6-20,6\% en los de $5 \mathrm{~cm}$ de diámetro, siendo los mayores valores para la mejor calidad de hormigón.

\section{BIBLIOGRAFIA}

1. A. HOFSOY: "Comparison of Apparent Compression Strength of Concrete Cores, Cubes and Cylinders". RILEM Symposium on the Experimental Research of Field Testing of Concrete. Trandheim, Noruega (1964).

2. N. PETERSONS: "Strength of Concrete in Finished Estructures". RILEM Symposium on the Experimental Research of Field Testing of Concrete. Trondheim, Noruega (1964).

3. F. CAMPUS, R. DUTRON y A. COUNE: "Controle de la qualite du beton en laboratoire et sur chantier». RILEM Symposium on the Experimental Research of Field Testing of Concrete. Trondheim, Noruega (1964)

4. R. JOOSTING: "Le prelevement et l'examen de petites carottes de beton". RILEM Symposium on the Experimental Research of Field Testing of Concrete. Trondheim, Noruega (1964).

5. A. PAVLIK: "Essais du beton durcissant dans les conditions de la construction.. RILEM Symposium on the Experimental Research of Field Testing of Concrete. Trondheim, Noruega (1964).

6. J. M. TOBIO: «Resistencia del hormigón en estructuras terminadas". INFORMES DE LA CONSTRUCCION, núm. 203 (1970).

7. J. L. RAMIREZ ORTIZ y J. M. BARCENA DIAZ: "Aportaciones en torno a la valoración de la calidad resistente del hormigón de estructuras por medio de probetas testigos extraídas por corten. INFORMES DE LA CONSTRUCCION, núm. 255 (1973).

8. P. JIMENEZ MONTOYA, A. GARCIA MESEGUER y F. MORAN CABRE: "Hormigón armado". Gustavo Gili, S. A. (1973).

9. «Instrucción para el proyecto y la ejecución de obras de hormigón en masa o armado». EH.73 (diciembre 1973).

10. J. L. RAMIREZ ORTIZ y J. M. BARCENA DIAZ: "Relación entre la resistencia del hormigón de las estructuras y la obtenida mediante ensayo de probetas testigo extraídas por corte". "Dyna", número 11 (noviembre 1974).

11. ADOLFO DELIBES LINIERS: "Análisis de la influencia de algunas variables en la extracción y ensayo a compresión de probetas-testigo de hormigón». INFORMES DE LA CONSTRUCCION, número 266 (diciembre 1974).

12. J. CALAVERA, G. APARICIO, A. DELIBES y G. GONZALEZ ISABEL: "El relleno de taladros producidos por la extracción de probetas-testigo y su influencia en la resistencia de los pilares de hormigón armado". INFORMES DE LA CONSTRUCCION, número 268 (febrero 1975). 


\section{résumé}

Evaluation de la résistance des poteaux en béton à l'aide d'éprouvettes: nouveaux apports

J. L. Ramírez Ortiz. Prof. Dr. ingénieur industriel

J. M. Bárcena Díaz, ingénieur industriel

Ce travail fait suite à celui qui a été publié dans le numéro 255 de cette revue, novem bre 1973, relatif au contrôle du béton durc à l'aide d'épan.

Tous les résultats obtenus aux différents essais sont exposés sous forme de tableaux et des graphiques. Pour les essais ont été
utilisés des poteaux de grandeur structurale.

Les sujets étudiés sont, en premier lieu, celui de la représentativité de la résistance des éprouvettes par rapport à celle du poteau dont celles-ci ont été prélevées et de la variation de cette résistance avec l'humidité et la hauteur du prélèvement sur la base du poteau.

En second lieu, les auteurs analysent la perte de résistance des poteaux, dont les trous, creusés pour le prélèvement d'une éprouvette de $10 \mathrm{~cm}$ de diamètre, sont bouchés, après cette opération, selon de différentes méthodes.

Les travaux de recherche ont été effectués aux laboratoires de la Chaire de Structures a l'Ecole Superieure des Ingénieurs Industriels de Bilbao et aux laboratoires d'essais et de recherche industrielle «L. J. Torrónteguin annexes de celle-ci.

\section{summary}

Evaluation of concrete pillars by means of drilled test samples: New contributions

J. L. Ramírez Ortiz, Prof. Dr. industrial engineering

J. M. Bárcena Díaz, industrial engineer

The present work is a continuation of that which appeared in this Journal, no. 255, NoThe total amount of results obtained in the
various tests are presented in the shape of various tests are presented in the shape of
tables and diagrams. For the tests pillars tables and diagrams. For the tests
of structural size have been used.

The studied subjects are resistance of the drilled specimens with regard to that of the column from which they are taken; and the variation of this resistance at changing humidity conditions and in relation to the extraction height above the column base.

The second subject to be analized is the resistance loss of the pillars, after the driled cores, with a diamer hove been extracted and after the resulting rious methods.

The research has been carried out in the aboratories of the Department of Structure of the College of Industrial Engineering in Bilbao and in the Testing and Industrial Research Laboratories "L. J. Torróntegui”.

\section{zusammentassung}

Auswertung des Widerstandes der Betonpfeiler mittelst gebohrter Probestücke: Neue Beiträge

J. L. Ramírez Ortiz, Prof. Dr. Gewerbebautechnik

J. M. Bárcena Díaz, Gewerbeingenieur

Die vorhandene Arbeit ist eine Forstsetzung der in dieser Zeitschrift, $n 0^{\circ} 255$, in November 1973, erschiedenen Arbeit, über das Thema: "Kontrolle des Festbetons mittels gebohrter Probestücke».

Die gesamten in den verschiedenen Proben erhaltenen Ergebnisse werden in linearen Darstellungen und Tabellen dargestellt. Fü die Proben sind Pfeiler von natüricher Baugrösse verwendet worden.

Die studierten Themen sind in erster Linie der Widerstand der gebohrten Probestücken im Verhälthis zu den Pfellern, denen sie entnommen worden sind, und ausserdem die Schwankungen dieses Widerstandes in Bezug auf Feuchtigkeit und Extrahierungshöhe über auf Feuchtigkeit und

Als zweites Thema wird der Widerstandsverlust der Pfeiler untersucht, deren nach der Probeentnahme (Durchmesser $10 \mathrm{~cm}$ ) entstandene Bohrlöcher durch verschiedene Methoden ausgefüllt worden sind.

Die Forschungsversuche sind in den Laboratorien der Bauabteilung der Technischen Hochschule für Gewerbeingenieure in Bilbao und in den dazugehörenden Laboratorien für Prüfungen und Industrieforschung aL. J. Torróntegui» unternommen worden.

\section{publicaciones del i. e. t. c. c.}

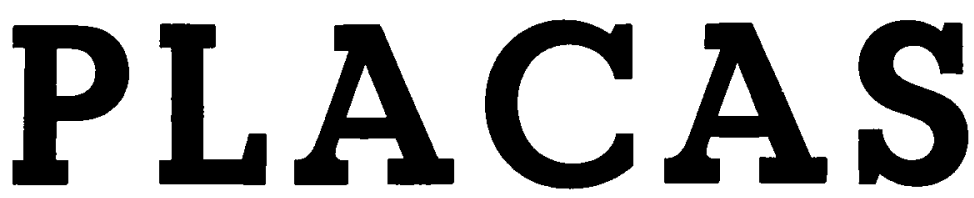

K. Striglat g H. Wippel

Drs. Ingenieros

Traducción de Juan Batanero

Dr. Ingeniero de Caminos

con la colaboración de

Francisco Morán

Ingeniero de Caminos

Este libro, cuidadosa y magníficamente editado, reúne, quizás, la más completa colección conocida de tablas para placas, por los numerosos casos de vinculación y de carga estudiados y por la abundancia de relaciones de dimensión y de datos ofrecidos, que cubren prácticamente todo el campo de las losas en edificación. Permite desarrollar, con comodidad, rapidez y una aproximación suficiente, los cálculos de dimensionamiento y comprobación, obviando las dificultades que como es sabido, presenta el desarrollo numérico de los métodos de cálculo de estos elementos, evitando enojosas operaciones.

Trata la obra sobre .Zonas de Placas», „Placas sobre apoyos puntuales», .Placas apoyadas en dos, tres y cuatro bordes y y Placas apoyadas elásticamente=, tipos que en la actualidad disponían de una documentación, incompleta o nula, para la determinación de esfuerzos. Los corrimientos de la placa, como valores previos para la determinación de los momentos, han sido obtenidos por medio del Cálculo de Diferencias, método que se ha comprobado como suficientemente satisfactorio, aún en su forma simple, aplicado con un cierto control.

Un volumen encuadernado en tela, de $30,5 \times 23,5 \mathrm{~cm}$, compuesto de 92 págs. Madrid, 1968.

Precios: España, 925 ptas.; extranjero, \$18.50. 